\title{
Estudo das pausas em idosos
}

\author{
Study of pauses in elderly
}

\author{
Vanessa de Oliveira Martins ${ }^{1}$, Claudia Regina Furquim de Andrade ${ }^{2}$
}

\begin{abstract}
RESUMO
Objetivo: Verificar a ocorrência de pausas na fala de idosos e sua distribuição quanto à frequência e a duração. Métodos: Participaram deste estudo 128 idosos de ambos os gêneros, divididos em dois grupos: GI (60-79 anos) e GII (a partir de 80 anos). Foram obtidas amostras de fala de todos os participantes para análise das rupturas de fala. As pausas foram classificadas como: hesitação (pausas de um a dois segundos) e pausa propriamente dita (acima de dois segundos). A análise computou 200 sílabas fluentes de cada amostra de fala. Os grupos foram comparados quanto às seguintes variáveis: presença de pausas e hesitações; duração média das pausas; porcentagem de tempo de pausas; frequência de pausas e hesitações; e frequência geral. Resultados: Para GII houve maior número de pausas e hesitações. Na análise da duração e da porcentagem de tempo de pausas não foi observada diferença entre os grupos. Em relação à frequência de pausas, hesitações e pausamento geral, observa-se diferença apenas para a última variável, com maior média de ocorrência para GII. Conclusão: Idosos com mais de 80 anos apresentam mais rupturas de fala por pausas que os idosos abaixo dessa idade. O estudo contribui como um balizador sobre as mudanças na fala relacionadas à quantidade de pausas e como um parâmetro comparativo para sintomas de doenças que se manifestam pelas variações de fala.
\end{abstract}

Descritores: Envelhecimento/fisiologia; Fala; Transtornos da linguagem/fisiopatologia; Memória; Idoso

\section{INTRODUÇÃO}

O envelhecimento da população é um fenômeno crescente em todo mundo e necessita ser mais estudado, a fim de assegurar melhor qualidade de vida para essa população $0^{(1)}$. Entretanto, estudos direcionados ao envelhecimento do processo de comunicação ainda são escassos, nessa população. O processo de envelhecimento pode ser acompanhado pelo declínio das capacidades tanto físicas, como cognitivas dos idosos, de acordo com suas características de vida ${ }^{(1)}$.

A partir dos anos 70, houve preocupação em se conhecer a fluência de fala ao longo da vida, já que o processo de envelhecimento causa mudanças biológicas e fisiológicas específicas, presbiacusia e ossificação das cartilagens da laringe, que afetam os mecanismos de fala e de audição no envelhecimento $^{(2)}$. Desta forma, seria razoável supor que tais mudanças pudessem influenciar a produção de fala, incluindo

Trabalho realizado no Departamento de Fisioterapia, Fonoaudiologia e Terapia Ocupacional, Faculdade de Medicina da Universidade de São Paulo - USP - São Paulo (SP), Brasil.

(1) Departamento de Fonoaudiologia, Faculdade de Medicina, Universidade Federal de Minas Gerais - UFMG - Belo Horizonte (MG), Brasil.

(2) Departamento de Fisioterapia, Fonoaudiologia e Terapia Ocupacional, Faculdade de Medicina, Universidade de São Paulo - USP - São Paulo (SP), Brasil.

Endereço de correspondência: Vanessa de Oliveira Martins. Faculdade de Medicina da Universidade Federal de Minas Gerais (UFMG), Departamento de Fonoaudiologia. Av. Prof. Alfredo Balena, 190, Santa Efigênia, Belo Horizonte (MG), Brasil, CEP: 30130-100. E-mail: vomartins@ufmg.br

Recebido em: 5/7/2010; Aceito em: 19/10/2010 os padrões de disfluências ${ }^{(2)}$.

No envelhecimento normal ocorre declínio das funções cognitivas relacionadas às habilidades verbais, visual-espaciais e temporais, em decorrência do processo de envelhecimento fisiológico natural dos lobos frontais ${ }^{(3,4)}$, observando-se efeito expressivo na produção de linguagem e não na compreensão ${ }^{(5)}$. Quanto à fala, as mudanças estão mais relacionadas à sua precisão, fluência, qualidade vocal e efetividade comunicativa. Tais mudanças podem ser similares àquelas que co-ocorrem em várias condições patológicas como disartria, apraxia, afasia, demência, dentre outras muito freqüentes entre os idosos ${ }^{(6)}$. Com o avançar da idade se manifestam muitos problemas relacionados aos aspectos perceptivo-motores da linguagem (como a audição, a fala e a voz) e aos aspectos propriamente linguísticos (como o acesso às palavras, a capacidade para compreender e produzir orações complexas, e para manejar diferentes formas de discurso) ${ }^{(7)}$. Estudo que buscava verificar os efeitos da hipertensão e diabetes mellitus sobre a habilidade de acesso lexical em idosos apontou efeito da hipertensão, que pode estar relacionado a mudanças microvasculares na região frontal do cérebro ${ }^{(8)}$. Desta forma, torna-se fundamental estudos com o objetivo de estabelecer parâmetros de normalidade de fala e linguagem para idosos saudáveis.

As alterações encontradas na memória operacional dos idosos produzem dificuldades para controlar o fluxo de informação e para regular e supervisionar os diferentes processos implicados em tarefas complexas, especialmente a linguagem ${ }^{(7)}$. Além disso, tais processos, parecem afetar seu componente articulatório, dando lugar a problemas de fluência verbal ${ }^{(9)}$. 
Em um estudo translinguístico, foi comprovado que nos adultos com mais de 70 anos pertencentes a diferentes culturas e línguas, acontece deterioração no rendimento linguístico geral que afeta diferentes níveis (fonético, morfológico, sintático, léxico e semântico) e processos linguísticos (compreensão, repetição, acesso ao vocabulário e construção de frases) ${ }^{(10)}$.

Estudos com idosos que apresentam alterações neuropsiquiátricas apontam dentre outras características, redução na velocidade de fala e aumento na duração e/ou tempo das pausas (relação entre a duração total de todas as pausas do discurso e a duração total da amostra de fala $)^{(11-14)}$, tornando-se importante se conhecer o padrão das pausas nos idosos saudáveis.

As habilidades de linguagem parecem estar afetadas de forma diferente na idade, algumas se mantêm intactas (compreensão de palavras, fala e outras atividades automáticas; compreensão de frases simples; tarefas que envolvam discriminação e reconhecimento; capacidade para narrar; fluência) enquanto outras se deterioram de modo evidente (nomeação e conversação; produção de sintaxe complexa; compreensão de sentenças complexas; tarefas que envolvam organização, iniciativa e planejamento; eleição de relevância em narrativas espontâneas; produtividade - taxa de informação - e precisão referencial; retenção de conteúdos e recordação imediata e tardia) $)^{(15)}$

O que mais chama a atenção na fluência do idoso é a ruptura, confundindo-se aspectos prosódicos com a própria organização do seu discurso. A descontinuidade de fala é um fenômeno normal na linguagem oral em qualquer faixa etária e nos idosos é marcada pelas rupturas, podendo ocorrer ao nível pragmático (descontinuidade do tema, pela interferência de segmentos parentéticos); sintático (pela ocorrência de frases interrompidas); léxico (pelas hesitações e truncamentos de vocábulos); e fonológico (pela presença de pausas) ${ }^{(16)}$. $\mathrm{O}$ excesso de pausas marca um ritmo construído aos arrancos. As pausas ocorrem em locais incomuns do enunciado, devido às hesitações provocadas pelas falhas de memória e pela incerteza do que dizer e como dizer ${ }^{(16)}$.

Em estudo anterior com o objetivo de verificar a variação da fluência da fala em idosos de 60 a 79 anos e idosos com mais de 80 anos, encontramos um aumento na nas taxas de rupturas de fala e uma diminuição na velocidade de fala ${ }^{(17)}$, corroborando as considerações encontradas na literatura ${ }^{(16)}$. Considerando o efeito do envelhecimento nas variáveis da fluência ao longo das décadas, observa-se variação mais sutil, sem diferenças significativas ${ }^{(17)}$.

No mesmo estudo pudemos observar que as únicas disfluências que diferenciaram os dois grupos foram as pausas e as hesitações, considerando-se as hesitações preenchidas (caracterizadas por vogais prolongadas) e as não preenchidas (pausas curtas de até dois segundos).

$\mathrm{O}$ objetivo do estudo aqui apresentado foi analisar as hesitações (pausas curtas de um a dois segundos) e pausas propriamente ditas (acima de dois segundos) na fala de idosos saudáveis.

\section{MÉTODOS}

Participaram desse estudo 128 idosos fluentes, falantes do
Português Brasileiro, de ambos os gêneros acima de 60 anos de idade, residentes na grande São Paulo, agrupados em: GI (idosos de 60 a 79 anos; n=84) e GII (idosos a partir de 80 anos; $\mathrm{n}=44$. Para este agrupamento foi utilizada a proposta do Medical Subject Headings (Index Medicus) $)^{(18)}$

Os critérios de inclusão dos participantes foram: nível sócio-econômico de classe média; ausência de queixa pessoal ou familiar de gagueira e/ou déficits de comunicação ou saúde associados; escore apresentado pelo Stuttering Severity Instrument (SSI) ${ }^{(19)}$ não indicativo de gagueira; ausência de histórico médico para uso crônico de medicação neuropsiquiátrica ou uso de drogas; ausência de histórico médico para presença de qualquer condição de déficit motor ou neurológico ou auditivo; ausência de histórico fonoaudiológico de distúrbio da comunicação.

Os participantes eram voluntários e foram selecionados em locais públicos, tais como praças, parques, igrejas, agências bancárias, dentre outros, localizados na Região Metropolitana de São Paulo.

Os procedimentos de seleção e avaliação dos participantes só foram iniciados após os processos éticos pertinentes: parecer da Comissão de Ética para Análise de Projetos de Pesquisa do Hospital das Clínicas da Faculdade de Medicina da Universidade de São Paulo - HCFMUSP (CAPEPesq n 1040/04) e assinatura do Termo de Consentimento Livre e Esclarecido pelo próprio participante.

Todos os participantes foram submetidos à coleta de amostra (filmada e gravada) de fala auto-expressiva a partir de figura e dada a seguinte ordem: "Por favor, olhe essa figura e me fale tudo que quiser sobre ela"(20). O discurso só foi interrompido por perguntas e/ou comentários, nos casos em que houve a necessidade de incentivar a produção do discurso para a obtenção de 200 sílabas expressas (fluentes), necessárias para análise da amostra ${ }^{(20)}$.

Os materiais utilizados para a coleta das amostras de fala foram: filmadora marca Panasonic ${ }^{\circledR}$ NVRJ36 fixada em tripé; fitas VHS-C; e cronômetro Nike® modelo WR0016-001, utilizado para a temporalização das amostras de fala.

As amostras de fala auto-expressivas foram transcritas literalmente, em sua totalidade, visando ao levantamento das rupturas de fala (hesitação, interjeição, revisão, palavra não terminada, repetição de palavra, repetição de segmento, repetição de frase, repetição de sílaba, repetição de som, prolongamento, bloqueio, pausa e intrusão de som ou segmento) de acordo com os critérios do Protocolo de Avaliação da Fluência da Fala ${ }^{(20)}$.

A análise das pausas incluiu as tipologias de hesitação (pausas curtas de um a dois segundos) e pausas propriamente ditas (acima de dois segundos). Para descrição das pausas foi utilizado o Protocolo para Análise de Pausas, elaborado para este estudo (Anexo 1). A análise foi dividida em cinco etapas: duração das pausas; porcentagem de tempo de pausas; frequência das pausas propriamente ditas; frequência das hesitações; e frequência geral.

- Duração das pausas: para essa análise todas as pausas das amostras de fala foram identificadas com um número, após isso foi verificada a duração de cada uma e realizada a marcação na coluna correspondente. Em seguida foi calculada a média de duração das pausas. 
- Porcentagem de tempo de pausas ${ }^{(11-14,21,22)}$ : mede o quanto o discurso é interrompido por pausas, refletindo aspectos cognitivos e emocionais dos parâmetros paralinguísticos da fala. Para o cálculo foi somada a duração de todas as pausas, medida a duração total da amostra e a aplicada a relação de porcentagem.

- Frequência das pausas (porcentagem em relação às rupturas de fala): mede a taxa de pausas propriamente ditas dentre às demais rupturas de fala. Para o cálculo foram contadas todas as pausas e rupturas e aplicada a relação de porcentagem.

- Frequência das hesitações (porcentagem em relação às rupturas de fala): mede a taxa de pausas curtas dentre às demais rupturas de fala. Para o cálculo foram somadas todas as hesitações e o total de rupturas e aplicada relação de porcentagem.

- Frequência geral: mede a taxa de pausamento (pausas mais hesitações) dentre às demais rupturas de fala. $\mathrm{O}$ calculo foi feito somando-se o número total de rupturas, após isso foi aplicada a relação de porcentagem.

Para análise estatística dos dados foi utilizado o teste nãoparamétrico de Mann-Whitney e o teste do Qui-quadrado. O nível de significância adotado foi de $5 \%$.

\section{RESULTADOS}

A média de idade de GI foi 70,19 anos (DP=5,47) e de GII foi 86,43 anos ( $\mathrm{DP}=5,05)$. Os grupos são homogêneos quanto à distribuição entre os gêneros $\left(X^{2}=0,415\right.$; n.g.l. $=1$; $\mathrm{p}=0,519)$.

Para a maioria dos participantes, em ambos os grupos, não houve a presença de pausas propriamente ditas (acima de dois segundos) nas amostras de fala (Tabela 1). Entretanto observase que a frequência de participantes de GII que apresenta tal ruptura é maior que em GI.

Tabela 1. Frequência de pausa propriamente dita

\begin{tabular}{lcccc}
\hline Variáveis & \multicolumn{2}{c}{ GI } & \multicolumn{2}{c}{ Gll } \\
\cline { 2 - 5 } & Frequência & $\%$ & Frequência & $\%$ \\
\hline Apresentaram pausa & 16 & 19,0 & 20 & 45,5 \\
Não apresentaram pausa & 68 & 81,0 & 24 & 55,5 \\
Total & 84 & 100 & 44 & 100 \\
\hline \multicolumn{3}{c}{$\left(\chi^{2}=9,961 ;\right.$ n.g.I. $\left.=1 ; p=0,002^{*}\right)$} \\
\hline
\end{tabular}

* Valores significativos $(p \leq 0,005)$ - Teste Qui-quadrado

Legenda: $\mathrm{GI}$ = grupo dos idosos de 60 a 79 anos; $\mathrm{Gll}=$ grupo dos idosos com mais de 80 anos
No caso das hesitações (Tabela 2), em ambos os grupos a frequência de idosos que apresenta tal ruptura é maior do que daqueles que não apresentam. Além disso, em GII a frequência de idosos que apresenta hesitações é maior que em GI.

Tabela 2. Frequência de hesitações

\begin{tabular}{lcccc}
\hline \multirow{2}{*}{ Variáveis } & \multicolumn{2}{c}{ Gl } & \multicolumn{2}{c}{ Gll } \\
\cline { 2 - 5 } & Frequência & $\%$ & Frequência & $\%$ \\
\hline Apresentaram hesitação & 51 & 60,7 & 38 & 86,4 \\
Não apresentaram & 33 & 39,3 & 6 & 13,6 \\
hesitação & 84 & 100 & 44 & 100 \\
Total & \multicolumn{3}{c}{$\left(\chi^{2}=8,967 ;\right.$ n.g.I.=1; $\left.p=0,003^{*}\right)$} \\
\hline
\end{tabular}

* Valores significativos ( $\mathrm{p} \leq 0,005)$ - Teste Qui-quadrado

Legenda: $\mathrm{Gl}=$ grupo dos idosos de 60 a 79 anos; Gll = grupo dos idosos com mais de 80 anos

Para análise da duração das rupturas (a frequência de pausas e da porcentagem do tempo de pausas) foram considerados apenas os participantes que apresentaram pausas propriamente ditas em suas amostras de fala ( $n=36$ idosos). Na Tabela 3 são apresentados os resultados da estatística descritiva e do teste de Mann-Whitney para as variáveis duração média das pausas e porcentagem de tempo de pausa, não sendo observada diferença significativa entre os grupos. $\mathrm{Na}$ análise da porcentagem de tempo de pausa foi encontrado valor discrepante em GI; entretanto a sua exclusão não alterou os resultados da análise. Desta forma, optamos por mantê-lo.

Para análise da frequência das hesitações foram incluídos apenas os participantes que as apresentaram em suas falas (n $\mathrm{GI}=51$; n GII=38) e para análise da frequência geral foram incluídos os idosos que apresentaram pausas e/ou hesitações (n GI=54; n GII=38).

A Tabela 4 mostra os resultados da análise estatística para as variáveis frequência de pausas; frequência de hesitações e frequência geral. Como pode ser observado, os grupos se diferenciam apenas para a frequência geral, ou seja, os idosos de GII apresentam ocorrência de pausas (pausas propriamente ditas mais hesitações) em relação às demais rupturas de fala estatisticamente maior que os idosos de GI.

\section{DISCUSSÃO}

Este estudo teve como objetivo caracterizar as pausas na fala de idosos e idosos com mais de 80 anos, quanto à ocorrência e à duração. De acordo com os resultados apresentados,

Tabela 3. Resultados da análise estatística para duração média das pausas e porcentagem de tempo de pausa

\begin{tabular}{|c|c|c|c|c|c|}
\hline Variáveis & Grupo & Média & Mediana & DP & Valor de $\mathrm{p}$ \\
\hline \multirow{2}{*}{ Duração média das pausas } & GI $(n=51)$ & 5,33 & 4,67 & 1,66 & \multirow{2}{*}{0,607} \\
\hline & GII $(n=38)$ & 5,26 & 4,71 & 2,29 & \\
\hline \multirow{2}{*}{ Porcentagem de tempo de pausas } & GI $(n=51)$ & 13,72 & 9,10 & 11,08 & \multirow{2}{*}{1,00} \\
\hline & GII $(n=38)$ & 14,94 & 13,08 & 11,08 & \\
\hline
\end{tabular}

* Teste Mann-Whitney $(\mathrm{p} \leq 0,005)$

Legenda: $\mathrm{GI}$ = grupo dos idosos de 60 a 79 anos; Gll = grupo dos idosos com mais de 80 anos; DP = desvio-padrão 
Tabela 4. Resultados da análise estatística para frequência de pausas, frequência de hesitações e frequência geral

\begin{tabular}{|c|c|c|c|c|c|}
\hline & Grupo & Média & Mediana & DP & Valor de $p$ \\
\hline \multirow{2}{*}{ Frequência de pausas } & $\mathrm{GI}(\mathrm{n}=54)$ & 12,04 & 9,10 & 7,79 & \multirow{2}{*}{0,886} \\
\hline & GII $(n=38)$ & 12,64 & 10,17 & 8,20 & \\
\hline \multirow{2}{*}{ Frequência de hesitações } & GI $(n=54)$ & 19,51 & 14,30 & 16,05 & \multirow{2}{*}{0,283} \\
\hline & GII $(n=38)$ & 24,29 & 16,67 & 18,66 & \\
\hline \multirow{2}{*}{ Frequência geral } & $\mathrm{GI}(\mathrm{n}=54)$ & 21,99 & 18,05 & 2,37 & \multirow{2}{*}{$0,019^{\star}$} \\
\hline & GII $(n=38)$ & 30,94 & 24,04 & 3,08 & \\
\hline
\end{tabular}

*Valores significativos $(p \leq 0,005)$ - Teste Mann-Whitney

Legenda: $\mathrm{Gl}$ = grupo dos idosos de 60 a 79 anos; $\mathrm{Gll}=$ grupo dos idosos com mais de 80 anos; $\mathrm{DP}=$ desvio-padrão

idosos mais velhos rompem a fala, por hesitação e pausa, com mais frequência.

Tais resultados estão de acordo com o encontrado em outro estudo com idosos falantes do Português Brasileiro ${ }^{(16)}$, que aponta que a fala dos idosos com mais de 80 anos é marcada pelo excesso de pausas. De acordo com tal estudo, as disfluências transmitem a sensação de insegurança, que parece ser a marca mais característica da fala dos idosos com mais de 80 anos. Além disso, com o avançar da idade as pausas tendem a aumentar, enquanto o tempo de articulação tende a decrescer, indicando que, no envelhecimento, não só os aspectos motores, mas também os cognitivos do comportamento falado tornam-se lentos.

Os dados de normalidade das variáveis relacionadas ao processamento motor da fala podem servir como parâmetro para o controle da evolução de doenças neuromotoras e neurodegenerativas ${ }^{(23)}$ como apresentado em estudo que verificou a progressão da doença de Parkinson pela análise de parâmetros de fala ${ }^{(24)}$. Além disso, podem ajudar no monitoramento de efeitos positivos ${ }^{(25)}$ ou negativos provocados por medicamentos que agem no sistema nervoso central em áreas relacionadas ao controle e processamento neuromotor da fala. Da mesma forma, poderão ser utilizados como indício de envelhecimento precoce ou para verificar se o processo de envelhecimento está mais acelerado que o esperado.

Estes resultados são importantes para os profissionais da saúde que trabalham com a população idosa. Para o fonoaudiólogo, podem servir para distinguir, com maior segurança, os processos de fala desviantes ${ }^{(17)}$, contribuindo para a promoção de saúde fonoaudiológica desta população, bem como para a melhora da qualidade de $\operatorname{vida}^{(1)}$.

Em estudo anterior realizado foram encontradas diferenças significativas para todas as variáveis da fluência pesquisadas (total de disfluências comuns, porcentagem de descontinuidade de fala e velocidade de fala), exceto para aquelas sugestivas de gagueira ${ }^{(17)}$. Em estudo longitudinal da população geral acima de sessenta anos, observou-se que nenhum dos participantes do estudo evidenciou um declínio generalizado em todas as habilidades cognitivas examinadas ${ }^{(26)}$. Além disso, o declínio desencadeado pelo envelhecimento incidiu, especialmente, nas tarefas que exigiam rapidez, atenção, concentração e raciocínio indutivo $^{(7,26)}$. Isto pode justificar porque no estudo anterior não se observa diferença nas variáveis da fluência ao longo das décadas. A diferença encontrada para pausas e hesitações no estudo citado ${ }^{(17)}$ e referendada no presente estudo pode estar relacionada à atenção e concentração para manter o tópico discursivo durante a conversa e a dificuldades relacionadas à memória também encontradas em indivíduos com mais de $80 \operatorname{anos}^{(1)}$.

Estudo longitudinal com indivíduos com idades entre 83 e 95 anos apontou que a fala foi uma das habilidades que se manteve preservada ao longo dos três anos do estudo e independente da escolaridade dos idosos ${ }^{(1)}$. De acordo com as autoras do estudo, as habilidades cognitivas ligada à linguagem são mais resistentes à degeneração pela idade, sendo por isso muito utilizada como indicador pré-mórbido na avaliação de condições cognitivas dos idosos. Por conseguinte, um desempenho prejudicado na área de linguagem tem sido utilizado como um marcador de uma demência em andamento ${ }^{(1)}$. No que tange a habilidades atencionais, observou-se que, no intervalo de três anos, os idosos apresentaram um decréscimo significativo no seu desempenho ${ }^{(1)}$. Os dados sugerem um prejuízo nas habilidades cognitivas de atenção e memória de trabalho ${ }^{(1)}$. Talvez o aumento na ocorrência de pausamento na fala de idosos de GII (mais de 80 anos), possa ser justificado porque falhas na memória episódica e operacional fazem com que durante tarefas discursivas os idosos percam a informação ou tenham dificuldades em acessá-las ${ }^{(7)}$, necessitando de mais tempo para o processamento. Tais considerações estão de acordo com outro trabalho que refere que dificuldade em atividades que exijam flexibilidade e velocidade mental durante o processamento de informações pode ser comumente observada durante o envelhecimento ${ }^{(27)}$.

Os resultados aqui apresentados apresentam restrições em decorrência do número de participantes do estudo não devendo ser generalizados, mas podendo contribuir como base para novas pesquisas. Outros estudos podem ser conduzidos relacionando o pausamento com medidas de memória e outros testes cognitivos. Além disso, seria interessante usar a análise acústica para identificação das pausas e da duração de cada uma delas e a hierarquia prosódica para se julgar a localização das mesmas.

\section{CONCLUSÃo}

A partir do estudo aqui apresentado, pode-se concluir que idosos com mais de 80 anos apresentam mais rupturas de fala por pausas que os idosos abaixo dessa idade. O estudo contribui como um balizador sobre as mudanças na fala relacionadas à quantidade de pausas e como um parâmetro comparativo para sintomas de doenças que se manifestam pelas variações de fala. 


\section{AGRADECIMENTOS}

Agradecemos à Fundação de Amparo à Pesquisa do Estado de São Paulo (FAPESP) e ao Conselho Nacional de Desen- volvimento Científico e Tecnológico (CNPq), pelo apoio concedido para realização dessa pesquisa, sob processo número 99/11163-9 e 142243/2005-1, respectivamente.

\begin{abstract}
Purpose: To verify the occurrence of pauses in the speech of elderly and their distribution according to frequency and duration. Methods: Participants were 128 elderly subjects of both genders, divided into two groups: GI (60-79 years) and GII (80 years and older). Speech samples were gathered from all participants for the analysis of speech disruptions. Pauses were classified as: hesitation (one to two seconds) and pause (over two seconds). The analysis considered 200 fluent syllables in each speech sample. The groups were compared regarding the following variables: presence of pauses and hesitations; average duration of pauses; percentage of pause time; frequency of pauses and hesitations; and general frequency. Results: There was a greater number of pauses and hesitations in GII. In the analysis of duration and percentage of pause time, no differences were found between groups. Regarding the frequency of pauses, hesitations and general pause (hesitation plus pauses), a difference was observed only for the last variable, with higher average occurrence for GII. Conclusion: Elderly over 80 years of age present more speech pauses than younger elderly. The study contributes as a marker on the changes in speech related to the amount of pauses, and as a comparative parameter for symptoms of diseases manifested by speech variations.
\end{abstract}

Keywords: Aging/physiology; Speech; Language disorders/physiopathology; Memory; Aged

\section{REFERÊNCIAS}

1. Argimon II, Stein LM. Habilidades cognitivas em indivíduos muito idosos: um estudo longitudinal. Cad. Saúde Pública. 2005;21(1):64-72.

2. Yairi E, Clifton NF Jr. Disfluent speech behavior of preschool children, high school seniors, and geriatric persons. J Speech Hear Res. 1972;15(4):714-9.

3. Grady CL, Craik FI. Changes in memory processing with age. Curr Opin Neurobiol. 2000;10(2):224-31.

4. Stebbins GT, Carrillo MC, Dorfman J, Dirksen C, Desmond JE, Turner DA, et al. Aging effects on memory encoding in the frontal lobes. Psychol Aging. 2002;17(1):44-55.

5. Tyler LK, Shafto MA, Randall B, Wright P, Marslen-Wilson WD, Stamatakis EA. Preserving syntactic processing across the adult life span: the modulation of the frontotemporal language system in the context of age-related atrophy. Cereb Cortex. 2010;20(2):352-64.

6. Kent RD. Research on speech motor control and its disorders: a review and prospective. J Commun Disord. 2000;33(5):391-427

7. Juncos $\mathrm{O}$, Pereiro AX. Comunicação e linguagem em idades avançadas. In: Puyuelo M, Rondal JA. Manual de desenvolvimento e alterações da linguagem na criança e no adulto. Porto Alegre: Artmed; 2007. p 479-97

8. Albert ML, Spiro A 3rd, Sayers KJ, Cohen JA, Brady CB, Goral M, Obler LK. Effects of health status on word finding in aging. J Am Geriatr Soc. 2009;57(12):2300-5.

9. Salthouse TA. Speed and knowledge as determinants of adult age differences in verbal tasks. J Gerontol. 1993;48(1):P29-36.

10. Juncos-Rabadán O, Iglesias FJ. Decline in the elderly's language: evidence from cross-linguistic data. J Neurolinguistics. 1994;8(3):18390.

11. Schalling E, Hammarberg B, Hartelius L. Perceptual and acoustic analysis of speech in individuals with spinocerebellar ataxia (SCA). Logoped Phoniatr Vocol. 2007;32(1):31-46.

12. Rosen KM, Kent RD, Delaney AL, Duffy JR. Parametric quantitative acoustic analysis of conversation produced by speakers with dysarthria and healthy speakers. J Speech Lang Hear Res. 2006;49(2):395-411.

13. Cannizzaro MS, Cohen H, Rappard F, Snyder PJ. Bradyphrenia and bradykinesia both contribute to altered speech in schizophrenia: a quantitative acoustic study. Cogn Behav Neurol. 2005;18(4):206-10.
14. Wang YT, Kent RD, Duffy JR, Thomas JE. Dysarthria in traumatic brain injury: a breath group and intonational analysis. Folia Phoniatr Logop. 2005;57(2):59-89.

15. Mansur LL, Radanovic M. Neurolinguística: princípios para a prática clínica. São Paulo: EI - Edições Inteligentes; 2004.

16. Preti D. A linguagem dos idosos: um estudo da análise da conversação. São Paulo: Contexto; 1991.

17. Andrade CR, Martins VO. Variação da fluência da fala em idosos. PróFono. 2010;22(1):13-8.

18. National Library of Medicine. Medical Subject Headings (Index Medicus) [Internet]. 2007 [cited 2007 Jun 12]. Available from: http:www.nlm.nih.gov/mesh/meshhome.htm.2007

19. Riley GD. Stuttering severity instrument for children and adults. Austin: Pro-Ed; 1994.

20. Andrade CR. Protocolo para avaliação da fluência da fala. Pró-Fono. 2000;12(2):131-4.

21. Stassen HH, Kuny S, Hell D. The speech analysis approach to determining onset of improvement under antidepressants. Eur Neuropsychopharmacol. 1998;8(4):303-10.

22. Cannizzaro M, Harel B, Reilly N, Chappell P, Snyder PJ. Voice acoustical measurement of the severity of major depression. Brain Cogn. 2004;56(1):30-5.

23. Martins VO. Variação da fluência da fala em falantes do português brasileiro: quatro estudos [tese]. São Paulo: Universidade de São Paulo, Faculdade de Filosofia, Letras e Ciências Humanas; 2007.

24. Skodda S, Rinsche H, Schlegel U. Progression of dysprosody in Parkinson's disease over time - a longitudinal study. Mov Disord. 2009;24(5):716-22.

25. Skodda S, Visser W, Schlegel U. Short- and long-term dopaminergic effects on dysarthria in early Parkinson's disease. J Neural Transm. 2010;117(2):197-205.

26. Schaie KW, Willis SL. Psychometric intelligence and aging. In: Blanchard-Fieds E, Hess TM, editors. Perspectives on cognitive change in adulthood and aging. New York: McGraw Hill; 1996. p. 293-322.

27. Souza VL, Borges MF, Vitória CM, Chiappetta AL. Perfil das habilidades cognitivas no envelhecimento normal. Rev CEFAC. 2010;12(2):186-92. 
Anexo 1. Protocolo para análise de pausas

Nome:

Data da avaliação:

1. Dados da Fluência da Fala

\begin{tabular}{|l|l|}
\hline Total de disfluências & \\
\hline Número de pausas & \\
\hline Número de hesitações & \\
\hline Duração da amostra de fala (em segundos) & \\
\hline
\end{tabular}

\section{Duração das pausas}

\begin{tabular}{|l|l|l|}
\hline Identificação & Duração (em segundos) & Média de duração \\
\hline 1 & & \\
\hline 2 & & \\
\hline 3 & & \\
\hline 4 & & \\
\hline 5 & & \\
\hline 6 & & \\
\hline 7 & & \\
\hline 8 & & \\
\hline 9 & & \\
\hline 10 & & \\
\hline
\end{tabular}

3. Porcentagem de tempo de pausa

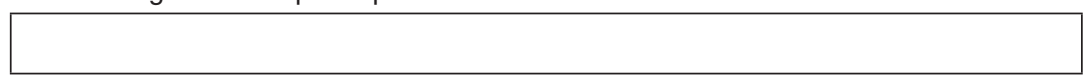

4. Frequência de pausamento

\begin{tabular}{|l|l|}
\hline Frequência de pausas & \\
\hline Frequência de hesitações & \\
\hline Frequência geral & \\
\hline
\end{tabular}

